\title{
Introduction to the Special Issue on the Mutual Shaping of Human-Robot Interaction
}

\author{
Somaya Ben Allouch ${ }^{1}$. Maartje de Graaf ${ }^{2}$. Selma Šabanović ${ }^{3}$
}

Published online: 9 August 2020

(c) Springer Nature B.V. 2020

Human-Robot interaction (HRI) research over the past decade has been shaped by many different disciplines, from social psychology to Artificial Intelligence, and by the broad range of theories and methodologies use in these fields. The field of HRI has, over time, also been able to develop and investigate robots in a plethora of challenging natural environments, in increasingly more complex scenarios and with diverse user groups. The many lines of inquiry in HRI research have enabled technological advancement and the resulting deployment of robots in natural interaction settings to realize (long term) human-robot interaction studies that are ecologically valid. The increasing variety of methodological perspective in HRI, including design thinking and ethnographic studies of robots in various contexts, gives a more holistic view on how (long-term) interactions between human and robots occur and what their consequences might be. These developments in HRI research fuel our idea that when robots enter different contexts of our everyday lives, they can shape and thus influence and change that particular context, while the context can lead to the use of robotic technology that goes beyond the purpose intended by its designers. The term mutual shaping guides our inquiry into the dynamic process of technological design and use suggesting that society and technology are not mutually exclusive but, instead, influence and shape each other. Societal institutions and practices shirt as a result of particular implementations of technology that has been created based on society's perceived needs and desires. The mutual shaping of technology and society approach focuses on analyzing how social and cultural factors influence the way technologies are designed, used, and evaluated, as well as

Maartje de Graaf

m.m.a.degraaf@uu.nl

1 Amsterdam University of Applied Sciences, Amsterdam, The Netherlands

2 University of Utrecht, Utrecht, The Netherlands

3 Indiana University Bloomington, Bloomington, USA how technologies affect our construction of social values and meanings.

The decisions made in the design, adoption, use, and evaluation process affect people's attitudes towards, uses of, and even their conceptualizations of (socially) interactive robotic systems. Social norms, values and morals are both implicitly and explicitly intertwined with technologies, reinforcing or altering our beliefs and practices. Once a robot has entered a social environment, it may lead people to alter the distribution of responsibilities and roles within that environment, including how people act in that situation or use context. This is an ongoing process in which the outcomes of human-robot interaction are influenced by both the social, technological and cultural context in which the interaction takes place. Accordingly, studies that show how use practices of robot systems and the social environment mutually shape each other, and what forms this mutual shaping process takes, are crucial for the future development of robots for broad societal use. This knowledge is required to inform the design and acceptance of new and existing robot systems and can have a transformational impact on (future) HRI research.

Our discussions of mutual shaping in HRI started with in 2016, with a workshop we organized on the topic at the annual RO-MAN conference in Lisbon. The papers featured in this special issue show their authors' own take on the notion of mutual shaping and seek to provide some examples of how the mutual shaping process between humans and robots takes place in different interaction environments and with different user groups.

In "Designing a social robot to support children's inquiry learning: A contextual analysis of children working together at school," Davison et al. identify a broad repertoire of social interactions and behaviors amongst school children to improve the learning experience of children supported by a social robot. Three main types of interactions where derived from their analysis: educational, collaborational and relational. Based on these interactions the authors present a set of design guidelines which inform the concrete robot behaviors to support the learning process of the children. 
Three papers address different aspects of mutual shaping in the domain of robots in healthcare. "Robotic Versus Human Coaches for Active Aging: An Automated Social Presence Perspective" by Martina Caic et al. addresses the challenge of elderly people's social perception of human versus robotic coaches in the context of an active and healthy ageing program. Hedonic and utilitarian value perceptions of exergames (i.e., video games integrating physical activity) were evaluated to assess elderly people's judgments of warmth and competence (i.e., social cognition) of their assigned coach which could be either a human or a robot. The authors demonstrate that socially assistive robots activate feelings of (automated) social presence in the elderly; that human coaches score higher on perceived warmth and competence relative to robotic coaches; and that social cognition impacts elderly people's experience of social robots and systems. In "Mutual Shaping in the Design and Use of Socially Assistive Robots: A Case Study on Social Robots for Therapy", Winkle et al. focus on a mutual shaping approach to the design of socially assistive robots. The authors propose the use case of social robots in therapy and present a methodology which combines elements of user-centered and participatory design with a focus on mutual learning in order to ensure successful deployment of socially assistive robots in therapy. In "The progressive intertwinement between design, human needs and the regulation of care technology: the case of lower-limb exoskeletons" Fosch Villaronga and Özcan introduce the notion that the current regulatory framework for care robot technology is ill-prepared to address multidisciplinary concerns because the authors argue that its primary focus is on physical safety requirements and it disregards other issues arising from the human-robot interaction. The authors emphasize the significant role design plays in shaping care robots to meet the needs of the users and the goals set by the regulation. To illustrate their argumentation, they use the example case of lower-limb exoskeletons. The authors conclude that regulation, design, and human needs should intertwine and mutually shape each other in HRI research.

Moving from the healthcare environment to public spaces, "Adaptive Side-by-side Social Robot Navigation to Approach and Interact with People" by Polo et al. present a new framework for how autonomous social robots approach and accompany people in urban environments for engendering better collaborations between people and robots. The authors develop a method which allows the robot, Tibi, to accompany a person by adapting the navigation of the robot in anticipation of future interactions with other people or contact with static obstacles. The outcomes of the social acceptability of the robot's performance of the accompanying, approaching and positioning tasks show that participants feel more comfort, creating a better experience of human-robot interaction. A second paper focusing on HRI in public spaces is 'Multi- ple Communication Roles in Human-Robot Interactions in Public Space.' In this paper, Fortunati et al. explore robots as media and the multiple communication roles they can play in public spaces and thus also shape the public space, all while being shaped by the public space in turn. The paper discusses three scenarios, the first is a human-robot interaction based on one-to-one or circular communication model; the second is a robot-human interaction based on a one-to many communication in presence; and the third is a robot-human interaction, based on the classical one-to-many communication model mediated through a television screen. This study shows that public patterns of behaviour toward the robot tend to be shaped by the ritualization of encounters between humans in the one-to-one communication model. The authors conclude that greater proximity and familiarity with the robot shapes the positive evaluations of the robot-human interaction.

Social robots need to operate in all kinds of different environments involving multiple users. In "Multi-party Turn-Taking in Repeated Human-Robot Interactions: An interdisciplinary Evaluation" Zarkowski argues that social robots that govern multi-user interactions require to be evaluated both from the technical and social standpoints. The author present an experiment involving the social robot EMYS participating in multi-party interaction where pairs of participants interacted with the robot in a trivia questions game lead by the robot.

The robot's multi-user interaction system was evaluated both in terms of technical performance and user assessment. By using both a technical and a social perspective this study showed that the robot was perceived by humans as more communicative, cooperative and fitting user expectations of the robot's actual behavior.

Finally, "The Social Pragmatics of Communication with Social Robots: Effects of Robot Message Design Logic in a Regulative Context" by Edwards et al. tackles the message design of social robots interacting with humans. The norms, values, and expectations with the process of communication itself shape important considerations for the interaction of the robot. Through the use of three different Message Design Logics, expressive, conventional, and rhetorical, in an online experiment the authors show that rhetorical message designs led to the most positive impressions of the robot by humans. This study illuminates the potential importance of social and cultural factors when analyzing the design and use of social robots in complex communication contexts and in forecasting the ways in which their social meanings and values may be construed.

HRI research aims to develop, apply, and evaluate knowledge about human-robot interaction in ever-more-challenging contexts and complex scenarios with diverse users in natural environments. By definition, the rapid developments currently occurring in the HRI field are by themselves shaped 
by internal and external social, technological, cultural, economic forces. As such, knowledge about the mutual shaping of humans and robots in real-life settings is central to the realization of an HRI research field which embraces different disciplines as its core. As a community we encounter new opportunities to work on research challenges to illustrate, including those showcased within this special issue, the deeply intertwined relations between social, technological and cultural factors that shape our current and further human-robot interactions. As guest editors, we hope that the articles featured in this special issue will inspire further research regarding the mutual shaping of human and robot interaction and foster exciting and productive interdisciplinary research findings. We wish to thank all of the contributors, reviewers, and editors without whom the realization of this special issue would not have been possible.

Publisher's Note Springer Nature remains neutral with regard to jurisdictional claims in published maps and institutional affiliations.

Somaya Ben Allouch is an Associate Professor and leading the Digital Life Research Group at the Amsterdam University of Applied Sciences. Her research expertise involves human-centred interaction, acceptance, (long term) use and evaluation of technologies. Specifically, social robotics, ubiquitous technologies, assistive technologies and wearables. She has been working from 2002 to 2014 at the University of Twente (the Netherlands) where she obtained her PhD in 2008 focusing on the development, acceptance and use of ambient intelligent technologies. She has been a visiting researcher at the Ambient Intelligence Research Lab of Stanford University in 2011 and 2012. She is a member of the editorial board of the Journal of Ambient Intelligence and Smart Environments and guest editor of the Journal of Distributed Sensor Networks. Furthermore, she is involved in various (inter)national competitively third-party funded research projects and supervises different $\mathrm{PhD}$ projects.
Maartje de Graaf is an Assistant Professor of Information and Computing Science at the University of Utrecht. Her research interest is on the intersection of interpersonal communication, social and cognitive psychology, and socially interactive technology with a focus on social robots. Envisioning a future in which the social abilities of robots can only increase, her research agenda evolves around people's cognitive, emotional, and behavioral responses to robots including the societal and ethical consequences of such responses. She has worked as a Postdoctoral Research Associate at Brown University's Humanity Centered Robotics Initiative (2017-2018) with a 2-year Rubicon grant from the Netherlands Organization for Scientific Research (NWO). She has a Bachelor of Business Administration in Communication Management (2005), a Master of Science in Media Communication (2011), and a PhD in Communication Science and Human-Robot Interaction (2015).

Selma Šabanović is an Associate Professor in the School of Informatics and Computing and the Cognitive Science Program and Director of the R-House Human-Robot Interaction Lab at Indiana University, Bloomington. Her work combines the social studies of computing, focusing particularly on the design, use, and consequences of socially interactive and assistive robots in different social and cultural contexts, with research on human-robot interaction (HRI) and social robot design. A common aim of her research is to go beyond studies of one-on-one interactions between people and robots to understand the effect of group-level, institutional, and cultural factors on HRI. Selma has been a Visiting Professor at Bielefeld University (2014), a lecturer in Stanford University (2008-2009), and a visiting scholar at AIST, Tsukuba, Japan and at Carnegie Mellon University (2005). She is an active participant in the social robotics and human-robot interaction communities, serving currently the General Chair of HRI 2018, and in prior years as the Program Committee chair for HRI 2016 and on the program committees of HRI, RO-MAN, ARSO, and ICSR conferences. Selma received her PhD in Science and Technology Studies from Rensselaer Polytechnic Institute in 2007. 